\title{
UNA COMBINACIÓN OBLIGADA EN SILENE DIVERSIFOLIA OTTH
}

\author{
Leopoldo MEDINA ${ }^{1 *}$, Carlos AEDO ${ }^{1}$, Marta FERNÁNDEZ-ALBERT $^{1}$ \& Joan PEDROL $^{2}$
}

Recibido el 9 de Julio de 2015, aceptado para su publicación el 15 de julio de 2015

A mandatory combination in Silene diversifolia Otth

Palabras clave. Caryophyllaceae, Silene diversifolia, Flora iberica, nomenclatura, Silene rubella.

Key words. Caryophyllaceae, Silene diversifolia, Flora iberica, nomenclature, Silene rubella.

Talavera (1990), en su tratamiento del género Silene L. en Flora iberica acepta el nombre de Silene rubella L. con dos subespecies: S. rubella L. subsp. rubella (Silene bergiana Lindm.; Silene rubella subsp. bergiana (Linndm.) Graebn. \& P. Graebn.) y S. rubella subsp. segetalis (Dufour) Nyman (Silene segetalis Dufour), la primera distribuida por el cuadrante suroccidental de la Península Ibérica y Marruecos, y la segunda extendida desde el S y E de la Península hasta Asia Menor.

Oxelman \& Lidén (1987: 477) habían realizado una propuesta de rechazo del nombre S. rubella debido a que el material tipo de Linneo corresponde en realidad $S$. bergiana. Esta propuesta fue posteriormente examinada por el Comité de Espermatófitos de la IAPT, que recomienda su aceptación (Brummitt, 1993: 687) y sugiere el uso del nombre Silene diversifolia Otth para el taxon mediterráneo, siguiendo la indicación de Oxelman (1991: 117) en su estudio del grupo. El rechazo definitivo del nombre $S$. rubella se produce en el Código de Viena (McNeil et al., 2006: apéndice V, apartado E).

Como parte de la actualización y mantenimiento de la información nomenclatural que tiene que ver con Flora iberica se han venido incluyendo en la página web del proyecto (www.floraiberica.es), en el epígrafe "Cambios nomenclaturales", aquellas modificaciones que han sido aceptadas e incluidas en los apéndices de las distintas ediciones de códigos de nomenclatura botánica (ICBN, ICN-AFP), como es esta referente a $S$. rubella de la que aquí nos ocupamos.

Para actualizar el tratamiento de S. rubella en la estructura nomenclatural de Anthos (2015) siguiendo las indicaciones del código de Viena y respetando el criterio taxonómico del autor del género en Flora iberica, hemos adoptado para este taxon el nombre de Silene diversifolia Otth con dos subespecies: a) $S$. diversifolia Otth subsp. diversifolia, para las plantas anteriormente tratadas como $S$. rubella subsp. segetalis y b) S. diversifolia subsp. bergiana, para aquellas tratadas como $S$. rubella subsp. rubella. Como quiera que no conocemos una combinación válida para esta última se hace necesario hacer la siguiente propuesta:

Silene diversifolia Otth subsp. bergiana (Lindm.) L. Medina, comb. nov.

$\equiv S$. bergiana Lindm. in Acta Horti Berg. 1(6): 3 (1891)

Este trabajo ha sido financiado por los proyectos Flora iberica (CGL2011-28613-C03-01) y Anthos (Fundación Biodiversidad y CSIC). 


\section{BIBLIOGRAFÍA}

ANTHOS -2015- Sistema de información de las plantas de España. Real Jardín Botánico, CSICFundación Biodiversidad. Recurso electrónico en www.anthos.es. Consulta realizada en junio de 2015.

BRUMMITT, R.K. -1993- Report of the Committee for Spermatophyta: 38. Taxon 42: 687-697.

MCNEILL, J., F.R. BARRIE, H.M. BURDET, V. DEMOULIN, D.L. HAWKSWORTH, K. MARHOLD, D.H. NICOLSON, J. PRADO, P.C. SILVA, J.E. SKOG, J.H. WIERSEMA \& N.J. TURLAND (eds.) -2006- International Code of Botanical Nomenclature (Vienna Code) adopted by the Seventeenth International Botanical Congress Vienna, Austria, July 2005. Gantner Verlag, Ruggell, Liechtenstein.
OXELMAN, B. \& M. LIDÉN -1987-Proposal to reject the name Silene rubella L. (Caryophyllaceae). Taxon 36: 477-478.

OXELMAN, B. -1991- Silene diversifolia Otth and related species in Europe: Bot. J. Linn. Soc. 106: 115-117.

TALAVERA, S. -1990- Silene L. In S. Castroviejo et al. (eds). Flora iberica 2: 313-406. Real Jardín Botánico, Madrid.

Dirección de los autores. 'Real Jardín Botánico, CSIC, Plaza de Murillo, 2, 28014 Madrid. ${ }^{2}$ Dept. Hortofruticultura, Botànica i Jardineria, Escola Tècnica Superior d'Enginyeria Agrària, Universitat de Lleida, Av. Alcalde Rovira Roure 191, 25198 Lleida.*Autor para correspondencia: medina@ rjb.csic.es 